\title{
STELLAR EVOLUTION, PARTICLE TRANSPORT, AND THE CP PHENOMENON
}

\author{
G. MICHAUD AND J. RICHER \\ ${ }^{1}$ Département de physique, Université de Montréal, Montréal, Canada, H3C 3.J \\ ${ }^{2}$ CERCA, Bureau 400, 5160 Boul. Décarie, Montréal, Canada \\ <michaudg@cerca. umontreal. ca〉, <richer@cerca. umontreal.ca〉
}

\section{From the Sun to Bp stars}

In the light of recent results from helioseismology, the abundance anomalies seen at the surface of $\mathrm{Bp}, \mathrm{Ap}, \mathrm{Am}$ and $\mathrm{Fm}$ stars appear as the measurable superficial effect of a process that has much more general impact.

It has recently been shown that He settling has a measurable impact on the solar pulsation spectrum. Indeed, now that very accurate helioseismological data are available, numerous recent models, (Bahcall et al. 1996; Basu et al. 1996; Guzik \& Cox 1992; Proffitt 1994; Richard et al. 1996; Guenther \& Demarque 1997), show that the calculated pulsation frequencies can be reconciled with observations only if the surface abundance of helium has decreased over time. The He abundance profile must also have changed. If helium sinks in the gravitational well, other massive elements are bound to do the same. Taking their diffusion into account improves agreement with the pulsation spectrum (Guenther \& Demarque 1997). Abundance anomalies caused by diffusion have become part of the new standard solar model (see also Turcotte et al. 1998).

It also appears that atomic diffusion plays a major role in $\mathrm{Bp}, \mathrm{Ap}, \mathrm{Am}$, and $\mathrm{Fm}$ stars where radiative acceleration leads to the formation of abundance anomalies (Michaud 1970). Atomic diffusion has been suggested to play a role in explaining the $\mathrm{Li}$ abundance gap that appears at $T_{\text {eff }} \simeq 6700 \mathrm{~K}$ in the Hyades and other clusters. Atomic diffusion might then affect most slowly rotating stars. The A and B peculiar stars are so the most evident members of a very large class. Understanding them is a necessary step in understanding stellar hydrodynamics.

\section{Atomic data, radiative accelerations and Rosseland opacities}

The relative abundances of the elements change during evolution when atomic diffusion is taken into account. If radiative forces are present there appear both over and underabundances. The models that, until now, have included atomic diffusion have neglected radiative accelerations and the local overabundances they can create within the star. A self consistent solution to the stellar evolution problem requires recalculating the Rosseland opacity, from monochromatic opacities of each important element, at the same time as the abundances evolve. OPAL and TOPbase (from the Opacity Project) have made available the atomic data required to calculate radiative forces throughout stellar models. In a current series of models (Turcotte et al. 1998; Richer et al. 1998), the opacities and radiative forces are continuously recomputed during evolution from OPAL's monochromatic data ( $\sim 1.5$ Gbyte of data in live memory) in order to include all effects of abundance changes due to diffusion and nuclear evolution.

\section{Application to Fm and Li-gap stars}

Solar models have been calculated to determine the mixing length and validate the evolution code. One surprising conclusion is that radiative accelerations can be up to $50 \%$ of gravity below the solar convection zone for iron peak elements. The same code was then used to calculate the evolution of $1.1,1.3,1.4,1.45$ and $1.5 \mathrm{M}_{\odot}$ stars. 

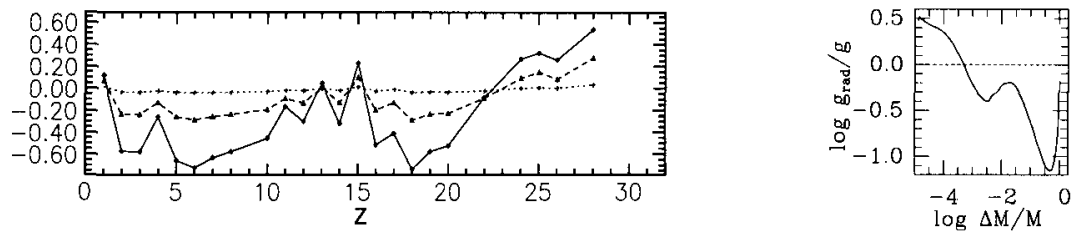

Figure 1. Left: evolution of surface abundances $\left(\log X / X_{0}\right)$ as a function of atomic number, in a $1.40 \mathrm{M}_{\odot}$ star. Dotted: after $70 \mathrm{Myr}$; dashed: $400 \mathrm{Myr}$; solid: $670 \mathrm{Myr}$. Right: radiative acceleration profile for $\mathrm{Ni}$ at $670 \mathrm{Myr}$, normalized to local gravity ( $\Delta M$ is the stellar envelope mass integrated from the surface).

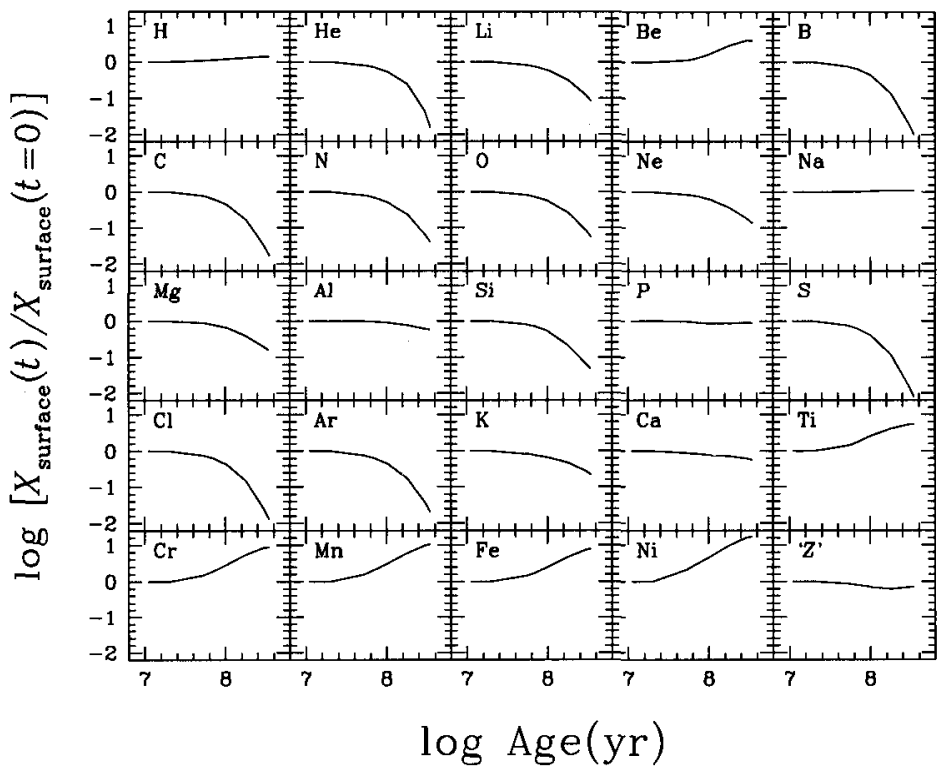

Figure 2. Evolution of surface abundances (mass fractions) of all the elements in a $1.45 \mathrm{M}_{\odot}$ stellar model.

Iron peak surface overabundances occur in stars more massive than $1.3 \mathrm{M}_{\odot}$. Local overabundances of iron peak elements increase the Rosseland opacity by factors of 3 to 6 which leads to a more massive convection zone. It is important to follow $\mathrm{Cr}, \mathrm{Mn}$, and $\mathrm{Ni}$ independently of $\mathrm{Fe}$, since they peak at different temperatures within the star.

In Figure 1 are shown results for the $1.4 \mathrm{M}_{\odot}$ model. At $t=670 \mathrm{Myr}$ this star has $T_{\text {eff }}=6693 \mathrm{~K}$ and $\log g=4.31$. The radiative acceleration for $\mathrm{Ni}$ is shown in the right hand panel. Similar $g_{\mathrm{rad}} \mathrm{s}$ were calculated for all the other elements included. The radiative acceleration is larger than gravity in the upper $0.1 \%$ of the mass. It increases again close to the center because the luminosity is more concentrated than the mass. The surface abundances obtained are shown at three time steps in the left hand panel as a function of the atomic number. Underabundances of $\mathrm{CNO}$ are clearly expected at the same time as those of $\mathrm{Li}$ and overabundances of iron peak elements.

Evolution of surface abundances is shown again for a slightly more massive star $\left(1.45 \mathrm{M}_{\odot}\right)$ in Figure 2. This model has evolved for only $350 \mathrm{Myr}$ and already variations by 2 orders of magnitude have developed (e.g., B, S, Cl). There are similarities with the previous case, but one notes that $\mathrm{Be}$ and $\mathrm{Na}$ are now supported, and $\mathrm{Al}$ and $\mathrm{P}$ are not, or just barely so. The abundances of some iron-peak elements are beginning to level off, which means that a region beneath the convection zone is now strongly depleted in these elements.

These abundances are to be compared to observations in F stars (Smith 1973; Burkhart \& 


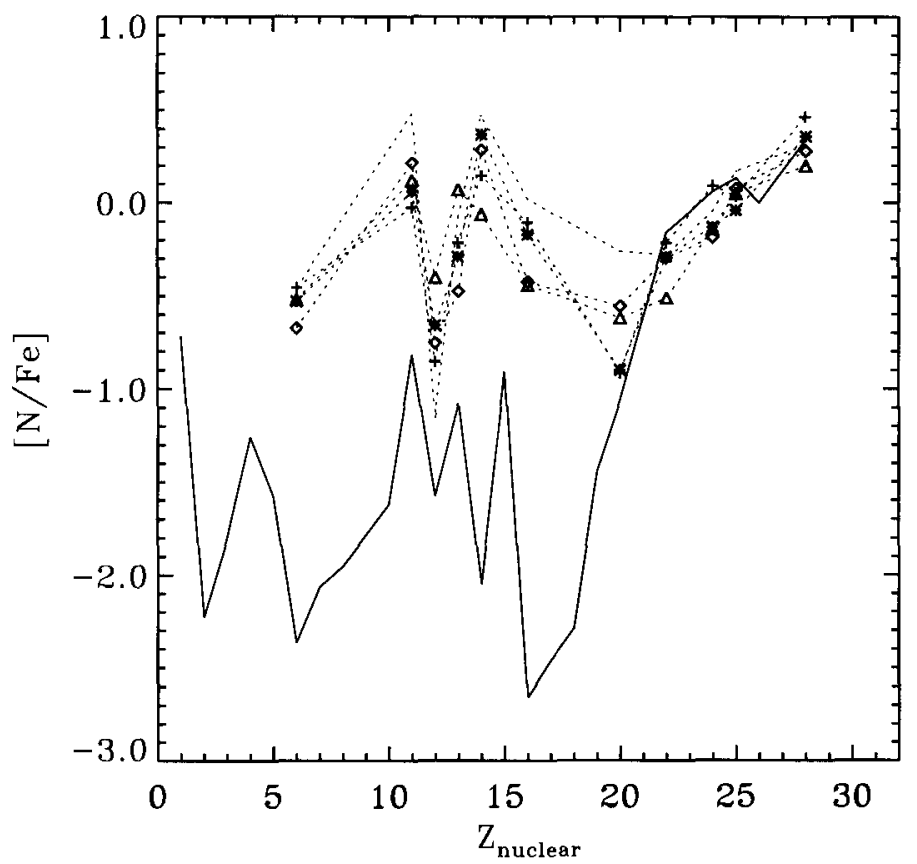

Figure 3. Predicted surface abundances at $300 \mathrm{Myr}$ (solid line) in a $1.45 \mathrm{M}_{\odot}$ main-sequence star, compared to observations by Smith (1973) (symbols) for slightly hotter Fm field stars.

Coupry 1991; Hui-Bon-Hoa \& Alecian 1997). There are similarities between the observed anomalies in the cooler stars and the calculated profiles. The observed anomalies are smaller than calculated and the $T_{\text {eff }}$ and gravity values do not exactly correspond. However, it seems clear that if diffusion were to explain entirely the Li gap, anomalies of metals larger than observed should occur at the same time.

In Fm stars (see Fig. 3), the observed abundances of elements with $Z=20$ to 28 , relative to the iron abundance, are very similar to the calculated values. The range of abundance anomalies is about 20. However the lower mass elements are observed to be much less underabundant than expected.

Composition evolution trends can change rapidly over time in more massive stars $\left(\sim 1.5 \mathrm{M}_{\odot}\right.$ and higher), for some elements, thus making comparisons to observations of stars of unknown age and mass very difficult. One may focus abundance studies on particularly well known stars, or else, simulate whole series of stars to produce abundance isochrones for each element. The sensitivity to stellar parameters of radiation induced surface abundance changes, is what makes such simulations so interesting, since they can become very useful diagnostic tools in the study of other aspects of stellar physics.

\section{Competing hydrodynamical processes}

The effects of atomic diffusion are likely to be reduced in most stars by other hydrodynamical processes. However the appropriate way to calculate this is by introducing atomic diffusion and the competing processes. Our approach is to obtain the evolutionary models with all effects of atomic diffusion first. These are the evolutionary models which can be calculated entirely from first principles, without arbitrary parameters other than the mixing length which is calibrated using solar models (Turcotte et al. 1998). They are the "standard" evolution models. Then one may introduce poorly understood physical processes, such as turbulence and mass loss, and test for 
their impact. Currently this requires the introduction of some arbitrary parameters; however there are now 28 abundances (and possibly more in the future) which could be measured and compared to calculated ones in order to constrain these parameters.

This research was partially supported at the Université de Montréal by NSERC.

\section{References}

Bahcall, J. N., Pinsonneault, M. H., Basu, S., \& Christensen-Dalsgaard, J. 1996, preprint

Basu, S., Christensen-Dalsgaard, J., Schou, J., Thompson, M. J., \& Tomczyk, S. 1996, ApJ, 460, 1064

Burkhart, C., \& Coupry, M.-F. 1991, A\&A, 249, 205

Guenther, D. B., \& Demarque P. 1997, ApJ, 484, 937

Guzik, J. A., \& Cox, A. N. 1992, ApJ, 381, 333

Hui-Bon-Hoa, A., \& Alecian, G. 1997, preprint

Michaud, G. 1970, ApJ, 160, 641

Proffitt, C. R. 1994, ApJ, 425, 849

Richard, O., Vauclair, S., Charbonnel, C., \& Dziembowski, W. A. 1996, A\&A, 312, 1000

Richer, J., Michaud, G., Rogers, F. J., Iglesias, C. A., Turcotte, S. \& LeBlanc, F. 1998, ApJ, in press

Smith, M. A. 1973, ApJS, 25, 277

Turcotte, S., Richer., J., Michaud, G., Iglesias, C.A. \& Rogers, F.J. 1998, submitted to ApJ 